\title{
Numerical and experimental analysis of wrinkling during the cup drawing of an AA5042 aluminium alloy
}

\author{
D. M. Neto ${ }^{1}$ (D) M. C. Oliveira ${ }^{1}$ - R. E. Dick ${ }^{2}$ - P. D. Barros ${ }^{1}$ • \\ J. L. Alves ${ }^{3}$ L. F. Menezes ${ }^{1}$
}

Received: 12 June 2015 / Accepted: 26 August 2015 /Published online: 4 September 2015

(C) Springer-Verlag France 2015

\begin{abstract}
The recent trend to reduce the thickness of metallic sheets used in forming processes strongly increases the likelihood of the occurrence of wrinkling. Thus, in order to obtain defect-free components, the prediction of this kind of defect becomes extremely important in the tool design and selection of process parameters. In this study, the sheet metal forming process proposed as a benchmark in the Numisheet 2014 conference is selected to analyse the influence of the tool geometry on wrinkling behaviour, as well as the reliability of the developed numerical model. The side-wall wrinkling during
\end{abstract}

D. M. Neto

diogo.neto@dem.uc.pt

M. C. Oliveira

marta.oliveira@dem.uc.pt

R. E. Dick

robert.dick@alcoa.com

P. D. Barros

pedro.barros@dem.uc.pt

J. L. Alves

jlalves@dem.uminho.pt

L. F. Menezes

luis.menezes@dem.uc.pt

1 CEMUC, Department of Mechanical Engineering, University of Coimbra, Polo II, Rua Luís Reis Santos, 3030-788 Pinhal de Marrocos, Coimbra, Portugal

2 Rigid Packaging Technology Division, Alcoa Technical Center, 100 Technical Drive, Alcoa Center, PA 15069, USA

3 MEMS, Microelectromechanical Systems Research Unit, University of Minho, Campus de Azurém, 4800-058 Guimarães, Portugal the deep drawing process of a cylindrical cup in AA5042 aluminium alloy is investigated through finite element simulation and experimental measurements. The material plastic anisotropy is modelled with an advanced yield criterion beyond the isotropic (von Mises) material behaviour. The results show that the shape of the wrinkles predicted by the numerical model is strongly affected by the finite element mesh used in the blank discretization. The accurate modelling of the plastic anisotropy of the aluminium alloy yields numerical results that are in good agreement with the experiments, particularly the shape and location of the wrinkles. The predicted punch force evolution is strongly influenced by the friction coefficient used in the model. Moreover, the two punch geometries provide drawn cups with different wrinkle waves, mainly differing in amplitude.

Keywords Side-wall wrinkling · Cup drawing - AA5042 aluminium alloy $\cdot$ Finite element method $\cdot$ Non-bifurcation analysis.

\section{Introduction}

Wrinkling, tearing and excessive springback are the major defects arising in sheet metal forming processes [1,2]. Due to aesthetic and/or functional reasons, wrinkling is typically undesired in finished sheet metal parts. Indeed, it is unacceptable in the automotive outer body panels, where the visual appearance is essential [3]. Furthermore, severe wrinkles may damage or even destroy the forming tools due to high contact pressures arising in the areas subjected to wrinkling [4]. However, the recent tendency in the automotive industry 
to use very thin high strength steel sheets to reduce fuel consumption and improve the safety, increases the likelihood of wrinkling during forming [5]. In the same way, the improvements carried out in the can manufacturing technology are aimed to reduce the weight and cost of the can bodies. Consequently, the new aluminium drawn and ironed beverage cans have been developed with thinner sidewalls and reduced base diameters, which also increases the probability of wrinkling occurrence [6].

The accurate prediction of wrinkling is extremely important in sheet metal forming to reduce as much as possible the expensive and time consuming "trial-and-error" procedures, as well as improving the quality of products. Wrinkling is a kind of local buckling of sheet metal that results from instability under compressive stresses [7]. According to Wang et al. [8], two types of wrinkling can occur during deep drawing of sheet metals: flange wrinkling and side-wall wrinkling, both generated from the compressive circumferential stresses. Since the cup wall is relatively unsupported by the tool, the compressive stress required to initiate the side-wall wrinkling is usually smaller than that for flange wrinkling [9]. However, the elimination of side-wall wrinkles by control of the blank holder force (changing the radial tensile stress component) is more difficult than the suppression of flange wrinkles [10].

Since the actual trend is to decrease the sheet thickness and increase the yield strength, wrinkling is becoming one of the primary failure modes in deep drawing processes. The initiation and growth of wrinkles are influenced by many factors such as mechanical properties of the sheet material, current stress state, tooling geometry, contact conditions including friction, and other process boundary conditions [11]. Several studies have been carried out to predict the occurrence, shape, and number of wrinkles. Nevertheless, these studies present a wide scattering of data for small deviations in the factors, as is common in instability phenomena [12]. The analytical methods provide the general tendency and the effect of individual parameters on the onset of wrinkling [13]. However, analytical approaches are restricted to relatively simple problems, and thus, cannot be employed in general sheet metal forming processes. Therefore, today wrinkling is mostly studied using the finite element method, which provides detailed information concerning the wrinkling behaviour of sheet metals in complex industrial problems. The buckling analysis with the finite element method can be performed using two different approaches: bifurcation analysis of perfect structure $[12,14]$ and non-bifurcation analysis $[15,16]$ considering an initial imperfection. Since all practical structures contain imperfections (material non-uniformity or geometric defects) typically the non-bifurcation analysis is more accurate than the bifurcation analysis [11].

Although there are evident advantages of the finite element simulation to investigate the wrinkling phenomena in sheet metal forming, the reliability of the numerical results is highly influenced by the simulation parameters, such as finite element type, mesh refinement, problems formulation and resolution, etc. The comparison between solid and shell elements in the prediction of the wrinkling pattern was carried out by Henriques et al. [17], showing the strong influence of the finite element formulation on the wrinkling onset and propagation. Considering only one layer of solid finite elements in the thickness direction, the predicted wrinkling pattern is in accordance with the experimental data, while the model using shell elements with five integration points along the thickness direction provides the wrong pattern. The effect of the finite element mesh size in the number of wrinkles arising in the cylindrical cup deep drawing process was investigated by Kim et al. [12]. When the height of the wrinkles is too small compared to the wavelength, the wrinkling behaviour cannot be accurately described using coarse meshes, requiring fine meshes in the regions where wrinkling is expected to occur [11]. Indeed, the prediction of wrinkling is very sensitive to the chosen mesh density.

The comparison between static explicit and dynamic explicit formulations was investigated by Kawka et al. [16] considering the deep drawing of conical cups. Comparing both formulations, the predicted wrinkling behaviour is different in terms of number, distribution and shape of wrinkles. A comparative study of static implicit and dynamic explicit procedures was performed by Kim et al. [18] for the wrinkling prediction in the hydroforming process. On one hand, they concluded that the dynamic effect in the explicit method plays an important role in the simulation results. A careful selection of the time and mass scaling leads to reasonable results when using the dynamic explicit approach. On the other hand, the adoption of the implicit scheme is more reliable and does not change the physics of the process, but requires high computational time because equilibrium is considered in each increment. Moreover, the initiation of the wrinkles without introducing an initial imperfection is difficult to predict using the static implicit approach, while the dynamic approach automatically generates deformed shapes with wrinkles due to the accumulation of numerical error [9].

The formability limit by onset of necking in sheet metal forming is typically determined based on the strain values in the forming limit diagram [19]. However, the wrinkling limit diagram cannot be defined by point variables such as strain or stress values since the wrinkling limit will also be highly influenced by tool geometry and contact conditions. The wrinkling limit diagram for anisotropic sheet metals subjected to biaxial plane-stress was proposed by Kim and Son [20] using bifurcation analysis. Nevertheless, the effects of sheet dimension on wrinkling behaviour were neglected with the assumption that wrinkling occurs locally [11]. The effect of plastic anisotropy on the puckering behaviour in the spherical cup deep drawing process was investigated by Anarestani et al. 
[4] using the bifurcation theory incorporated in the incremental finite element analysis. The results show that the plastic buckling tendency decreases while the normal anisotropy increases. The planar anisotropy plays a role of imperfection, inducing the number of predicted wrinkle waves.

In the present study, the wrinkling behaviour of a sheet metal forming process is analysed by the finite element method using the non-bifurcation analysis and compared with experimental measurements. The influence of the tool geometry, the material constitutive model and the friction conditions on the side-wall wrinkling is investigated considering the benchmark 4 proposed in Numisheet 2014 - Wrinkling during cup drawing. The organization of the paper is the following. The experimental procedure of the cylindrical cup drawing is described in Section 2, as well as the approach used to measure the wrinkles. The developed finite element model is presented in Section 3, including a mesh sensitivity analysis for the blank. The comparison between numerical predictions and experimental results is carried out in Section 4, showing the accuracy of the numerical solution. The main conclusions of this study are discussed in Section 5.

\section{Experimental procedure}

The benchmark 4 proposed in Numisheet 2014 was selected for this investigation, which was designed to study the wrinkling occurrence during the cylindrical cup drawing process [21]. The main objective of this study is to investigate the effects of tool geometry and the material constitutive model of the sheet on dome wrinkling (puckering) behaviour. The cup drawing operation involves three axisymmetric forming tools (die, blank-holder and punch), which are schematically illustrated in Fig. 1, as well as their main dimensions in millimetres. In order to investigate the effects of geometry on the wrinkling behaviour, two different punch geometries are studied, where the difference between them is simply defined by the dimension $h$ shown in Fig. 1 (b). Consequently, two distinct process conditions are defined, the so-called condition $A$ which involves a punch with $h=5.207 \mathrm{~mm}$, and the so-called condition $C$ for which $h=3.429 \mathrm{~mm}$. The circular blank of an AA5042 aluminium alloy with $64.77 \mathrm{~mm}$ of diameter and $0.2083 \mathrm{~mm}$ of initial thickness is considered in this benchmark.

The deep drawing of the cylindrical aluminium cups is carried out at room temperature in a hydraulic press with maximum capacity of 5 tonnes using the set of forming tools schematically shown in Fig. 1. The press is equipped with a monitoring system (Keithley), which enables the acquisition of the punch force evolution as a function of its stroke and the blank-holder force. The experimental tests are run in triplicate for each process condition in order to investigate the reproducibility. The drawing process is performed with a constant punch travel speed of $140 \mathrm{~mm} / \mathrm{s}$ and a constant blank-holder force of $8.9 \mathrm{kN}$. The cup is drawn completely considering a punch travel of $20 \mathrm{~mm}$, while the gap between the blankholder and the die is not restricted by any rigid stop during the drawing process. All forming tools are made of AISI A2 steel [22] with 58-60 of Rockwell C hardness and surface roughness about 2-4 $\mu \mathrm{m}$ (finish working surfaces). An oil lubricant is applied to both sides of the blank to reduce the frictional forces between the forming tools and the blank sheet during the experiments. The final geometry of the cylindrical aluminium drawn cup, performed under the condition A, is presented in Fig. 2, highlighting the development of wrinkles in the conical sidewall of the drawn cup [21].

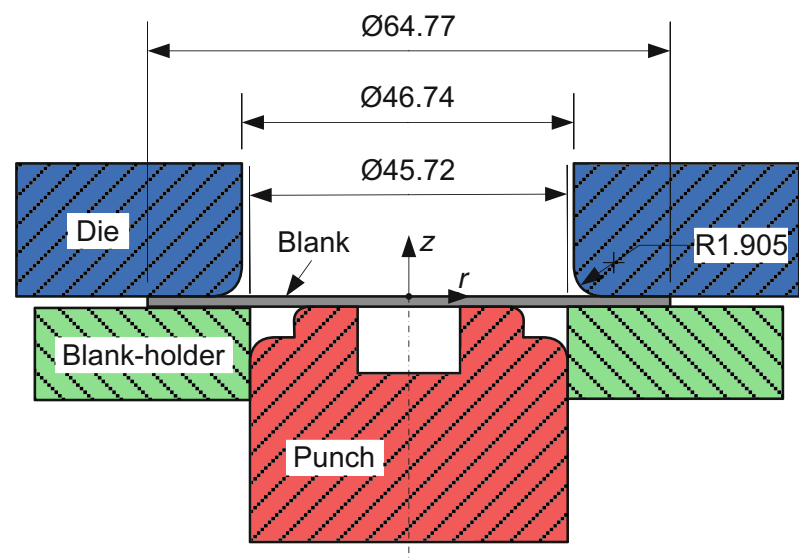

(a)

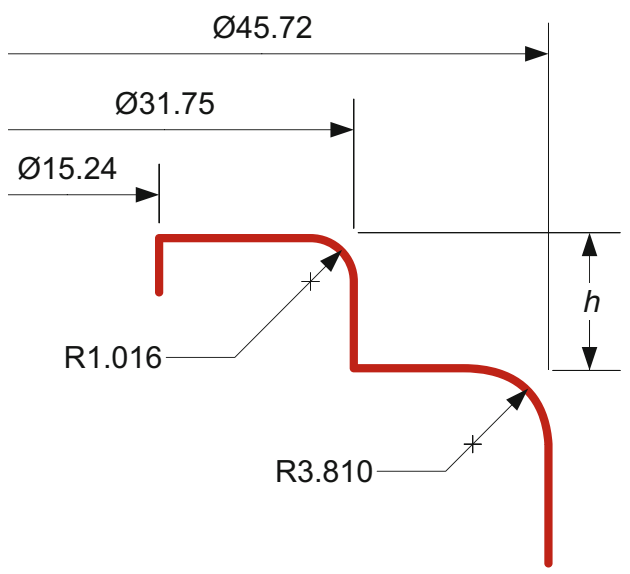

(b)

Fig. 1 Schematic of the forming tools used in the drawing process and their dimensions (mm): (a) global view; (b) detail view of the punch geometry with the variable dimension $h$ 


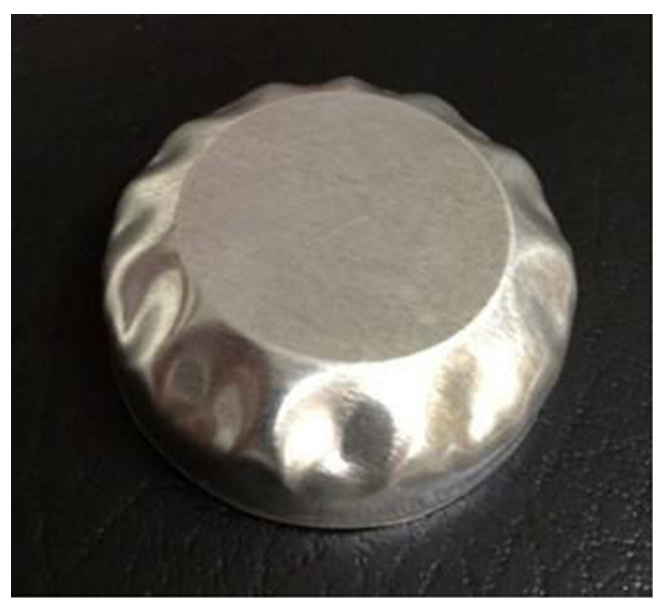

Fig. 2 Development of side-wall wrinkles in the experimental drawing of a cylindrical cup [21]

The wrinkling behaviour during the drawing is defined in this study by the wrinkle waves developed along the unsupported side-wall area of the cup [21]. The wrinkle contours are measured in the outer surface of the drawn cup using a coordinate measuring machine (CMM). The geometrical measurements are performed at several circumferential paths (layers) with increments of $0.5 \mathrm{~mm}$ along the $z$-direction (negative) from the top surface of the drawn cup. The radial distance of the points is calculated with respect to the cup axis, which is evaluated along the circumferential direction specified by the rolling direction. The processed CMM scan data provides the amplitude and frequency of the wrinkle pattern for both conditions studied (punches with different profile depths).

\section{Finite element model}

Since side-wall wrinkling is expected to occur during the axisymmetric deep drawing problem, a 3D finite element model is mandatory for an accurate analysis. The numerical simulations of the cup drawing process are performed with the in-house static implicit finite element code DD3IMP [23], which has been specifically developed to simulate sheet metal forming processes [24]. The kinematic description of the blank is described by an updated Lagrangian formulation. The forming tools are considered rigid bodies with its surface modelled by Nagata patches [25], while the nodal normal vectors required for the contact surface smoothing approach are evaluated through the IGES file using the algorithm proposed by Neto et al. [26]. The friction between the blank and the forming tools is described by the classical Coulomb's law. The frictional contact problem is regularized through the augmented Lagrangian method [27], leading to a mixed system of equations involving both displacements and contact forces as unknowns. An explicit approach is used to calculate an approximate first solution for the nodal displacements, the stress states and frictional contact forces. Then, in order to improve the convergence during the subsequent iterative stage, the increment size (defined by the user) is adjusted using a generalization of the $r_{\min }$ strategy proposed by Yamada et al. [28]. The first trial solution is iteratively corrected using the generalized Newton method until a satisfactory equilibrium state of the deformable body is achieved. In order to improve computational performance, some high performance computing techniques have been incorporated in DD3IMP to take advantage of multi-core processors, namely OpenMP directives in the most time consuming branches of the code [29].

Due to geometric and material symmetry conditions of the cup drawing process, only one quarter of the model is simulated. The blank is discretized with 8-node hexahedral finite elements associated with a selective reduced integration scheme [30]. The modelling of the blank with solid elements allows the accurate evaluation of the contact forces and the through-thickness gradients (stress and strain) during deformation in the can-making process [31]. The friction coefficient between sheet and tools is assumed to be constant and taken from the benchmark specifications as $\mu=0.03$. The blank geometry (one-quarter) is divided in two zones, as shown in Fig. 3. The central zone of the blank (radius inferior to $12.5 \mathrm{~mm}$ ), which corresponds to the flat area of the punch (see Fig. 1), is characterized by small strains during the drawing process. Therefore, it is discretized by a relatively coarse unstructured mesh. On the other hand, the blank zone in which wrinkling is expected to occur is discretized with a fine structured mesh to accurately reproduce the wrinkling waves. In the present study, the finite element model does not contain

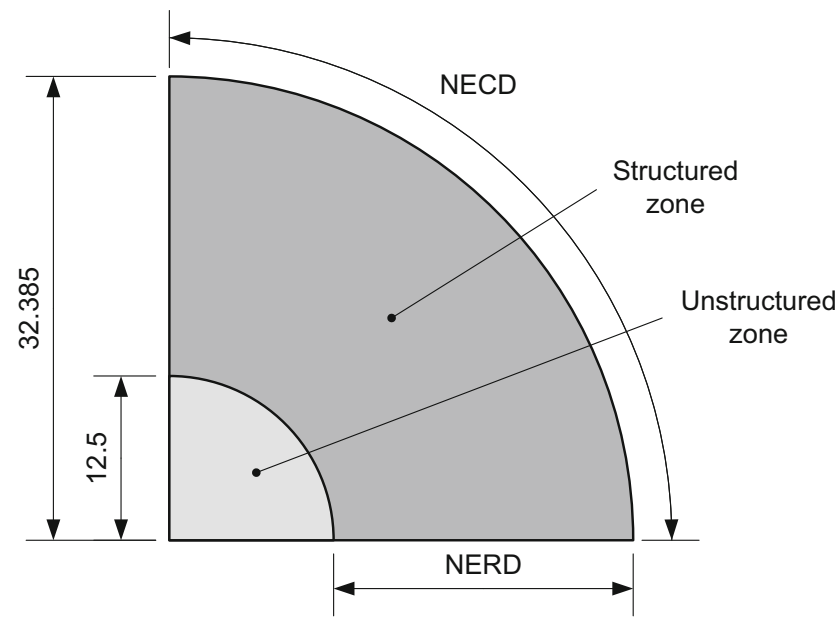

Fig. 3 Division of the blank in two zones to perform a structured mesh on the wrinkle region (NERD: number of elements in the radial direction; NECD: number of elements in the circumferential direction, dimensions in $\mathrm{mm}$ ) 
Table 1 Elastic proprieties and material parameters of the work hardening law

\begin{tabular}{ll}
\hline Elastic properties & Isotropic hardening (Voce law) \\
\hline$E=68,900 \mathrm{MPa}$ & $Y_{0}=267.8 \mathrm{MPa}$ \\
$\nu=0.33$ & $Y_{\text {sat }}=375.1 \mathrm{MPa}$ \\
& $C=17.86$ \\
\hline
\end{tabular}

any initial imperfection to induce wrinkling. In fact, the initiation of the wrinkles arises as results of the round-off error present in the finite element simulation.

\section{Material constitutive behaviour}

The mechanical behaviour of the AA5042 aluminium alloy is assumed to be elastic-plastic. The elastic behaviour is considered isotropic and constant, which is defined by the Young's modulus and the Poisson's ratio indicated in Table 1. The characterization of the plastic response requires the specification of a hardening law, a yield function and a flow rule by which the subsequent plastic deformations can be calculated. The plastic strain increment vector is normal to the yield surface when the associated flow rule is considered in the orthotropic behaviour description. The isotropic work hardening behaviour is modelled by the Voce law:

$Y=Y_{\text {sat }}-\left(Y_{\text {sat }}-Y_{0}\right) \exp \left(-C \bar{\varepsilon}^{\mathrm{p}}\right)$,

where $Y$ is the flow stress, and $\varepsilon^{\mathrm{p}}$ denotes the equivalent plastic strain, while $Y_{0}$ (initial yield stress), $Y_{\text {sat }}$ and $C$ are material parameters according to the Voce law. The kinematic hardening is not taken into account in this study since the experimental tests required to perform the parameters identification are not available (e.g. Bauschinger tests). The material parameters

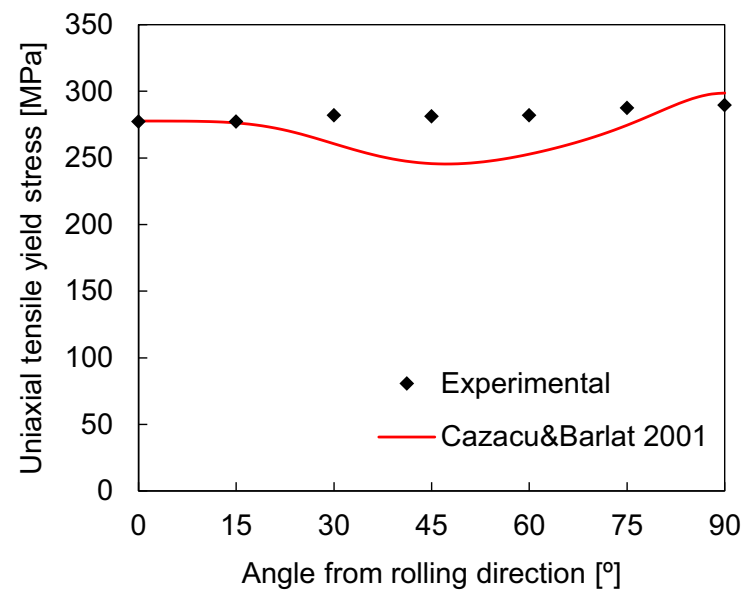

(a)
Table 2 Material anisotropy parameters of the Cazacu\&Barlat 2001 yield criterion

\begin{tabular}{lllllllll}
\hline$a_{1}$ & $a_{2}$ & $a_{3}$ & $a_{4}$ & $a_{5}$ & $a_{6}$ & $b_{1}$ & $b_{2}$ & $b_{3}$ \\
\hline 0.838 & 0.981 & 1.242 & 1.252 & 1.000 & 1.000 & 37.49 & 9.458 & 31.94 \\
$b_{4}$ & $b_{5}$ & $b_{6}$ & $b_{7}$ & $b_{8}$ & $b_{9}$ & $b_{10}$ & $b_{11}$ & $c$ \\
8.142 & -18.08 & 1.000 & 1.000 & 1.000 & 1.000 & 15.89 & 1.000 & 0.004
\end{tabular}

describing the isotropic work hardening law are evaluated by minimizing the relative difference between experimental and numerical stress-strain curves of the uniaxial tensile test oriented along the rolling direction [21]. The three parameters of the Voce law obtained from the optimization procedure are presented in Table 1.

An accurate description of the anisotropy in the rolled sheets is essential for reliable simulations of cup drawing process, since it governs the material flow characteristics during forming operation [32]. Phenomenological plasticity theories consider initial anisotropy through a yield criterion. Although widely used for anisotropic materials, the Hill' 48 yield criterion is known to inaccurately describe the behaviour of materials with low $r$-values, such as aluminium alloys [33]. Therefore, the advanced yield criterion proposed by Cazacu and Barlat [34] is adopted in this study to model the anisotropic behaviour of the AA5042 aluminium alloy. It is based in the generalization of the invariants of the deviatoric stress tensor to orthotropy. These generalized stress invariants are then used to extend the Drucker's isotropic yield criterion to orthotropy, such that:

$Y=\left\{27\left(\left(J_{2}^{0}\right)^{3}-c\left(J_{3}^{0}\right)^{2}\right)\right\}^{1 / 6}$,

where $J_{2}^{0}$ and $J_{3}^{0}$ are the second and third generalized invariants of the effective deviatoric stress tensor.

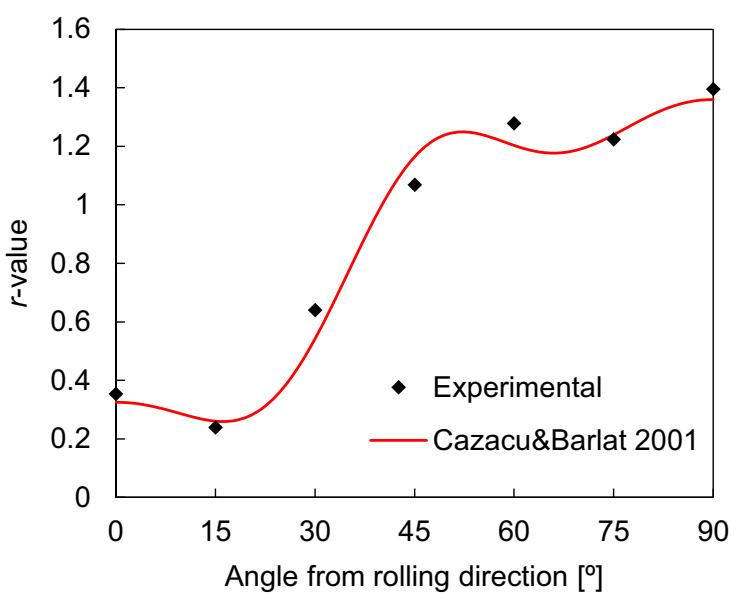

(b)

Fig. 4 Material anisotropy of the aluminium alloy AA5042 described by the Cazacu\&Barlat 2001 yield criterion compared with experimental values: (a) uniaxial tensile yield stress; (b) plastic strain ratio 
Table 3 Number of finite elements used in each zone of the blank for three different meshes (2 layers of elements through the thickness). RD: radial direction

\begin{tabular}{llllllll}
\hline & NERD & Element length in RD [mm] & NECD & Element central angle $\left[{ }^{\circ}\right]$ & Structured zone & Unstructured zone & Total of elements \\
\hline Mesh \#1 & 100 & 0.199 & 100 & 0.90 & 20,000 & 2546 & 22,546 \\
Mesh \#2 & 120 & 0.166 & 200 & 0.45 & 48,000 & 3802 & 51,802 \\
Mesh \#3 & 150 & 0.132 & 250 & 0.36 & 75,000 & 4630 & 79,630 \\
\hline
\end{tabular}

The second invariant generalized to orthotropy is defined as:

$$
\begin{aligned}
J_{2}^{0}= & \frac{a_{1}}{6}\left(\sigma_{x x}-\sigma_{y y}\right)^{2}+\frac{a_{2}}{6}\left(\sigma_{y y}-\sigma_{z z}\right)^{2}+\frac{a_{3}}{6}\left(\sigma_{x x}-\sigma_{z z}\right)^{2} \\
& +a_{4} \sigma_{x y}^{2}+a_{5} \sigma_{x z}^{2}+a_{6} \sigma_{y z}^{2},
\end{aligned}
$$

where the coefficients $a_{i}(i=1 \ldots 6)$ reduce to unity in the isotropic case. The third invariant generalized to orthotropy is defined as:

$$
\begin{aligned}
J_{3}^{0}= & \frac{1}{27}\left(b_{1}+b_{2}\right) \sigma_{x x}^{3}+\frac{1}{27}\left(b_{3}+b_{4}\right) \sigma_{y y}^{3}+\frac{1}{27}\left(2\left(b_{1}+b_{4}\right)-b_{2}-b_{3}\right) \sigma_{z z}^{3} \\
& -\frac{1}{9}\left(b_{1} \sigma_{y y}+b_{2} \sigma_{z z}\right) \sigma_{x x}^{2}-\frac{1}{9}\left(b_{3} \sigma_{z z}+b_{4} \sigma_{x x}\right) \sigma_{y y}^{2} \\
& -\frac{1}{9}\left(\left(b_{1}-b_{2}+b_{4}\right) \sigma_{x x}+\left(b_{1}-b_{3}+b_{4}\right) \sigma_{y y}\right) \sigma_{z z}^{2} \\
& +\frac{2}{9}\left(b_{1}+b_{4}\right) \sigma_{x x} \sigma_{y y} \sigma_{z z}-\frac{\sigma_{x z}^{2}}{3}\left(2 b_{9} \sigma_{y y}-b_{8} \sigma_{z z}-\left(2 b_{9}-b_{8}\right) \sigma_{x x}\right) \\
& -\frac{\sigma_{x y}^{2}}{3}\left(2 b_{10} \sigma_{z z}-b_{5} \sigma_{y y}-\left(2 b_{10}-b_{5}\right) \sigma_{x x}\right) \\
& -\frac{\sigma_{y z}^{2}}{3}\left(\left(b_{6}+b_{7}\right) \sigma_{x x}-b_{6} \sigma_{y y}-b_{7} \sigma_{z z}\right)+2 b_{11} \sigma_{x y} \sigma_{x z} \sigma_{y z}
\end{aligned}
$$

where the coefficients $b_{i}(i=1 \ldots 11)$ reduce to unity for isotropic conditions. This yield criterion involves 18 material

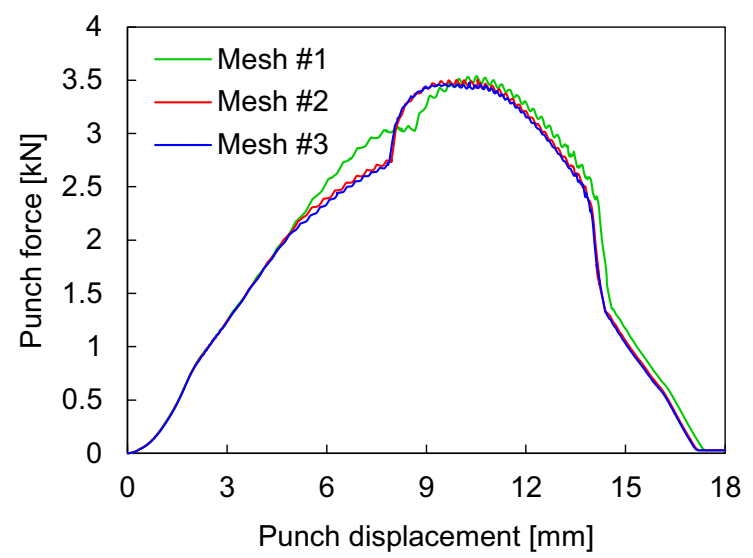

(a) parameters for 3D stress conditions. For metal sheets the parameters $a_{5}, a_{6}$ and $b_{k}(k=6,7,8,9,11)$ cannot be evaluated, thus the corresponding isotropy condition values are commonly adopted, i.e. 1.0. Therefore, the anisotropy parameters reduce to 11 , which are: $a_{k}(k=1, \ldots, 4), b_{k}(k=10)$ and $c$.

Due to the large amount of anisotropy parameters involved in the definition of this advanced yield criterion (Cazacu\&Barlat 2001), the parameter identification procedure becomes complex [35]. In the present study it was performed using the in-house code DD3MAT [36] specifically developed for this purpose, which is based on the minimization of an error function that evaluates the difference between the estimated values and the experimental ones. However, the conditions that guarantee the convexity of Cazacu\&Barlat 2001 are unknown. Therefore, following Soare et al. [37], the minimization process adopted includes testing the convexity of the yield surface for several planes in the stress space [38]. The experimental data provided by the benchmark committee [21] for the AA5042 aluminium alloy comprises the initial tensile yield stresses and the plastic strain ratio (Lankford coefficient) values for seven equally spaced orientations with the rolling direction (see Fig. 4). The normal anisotropy coefficient of this aluminium alloy is $r=\left(r_{0}+2 r_{45}+r_{90}\right) / 4=0.972$, while the planar anisotropy coefficient is given by $\Delta \mathrm{r}=\left(r_{0}-2 r_{45}+r_{90}\right) / 2=-0.194$, where the subscripts refer to the angle between the tensile specimen axis and the rolling direction of the sheet.

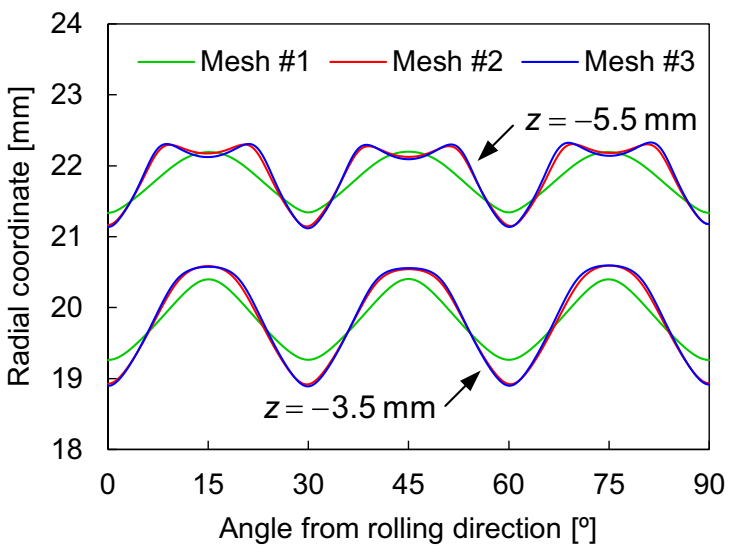

(b)

Fig. 5 In-plane mesh refinement considering material isotropy (condition A): (a) punch force evolution; (b) radial coordinate of the side-wall cup evaluated in two layers as function of the angular position 


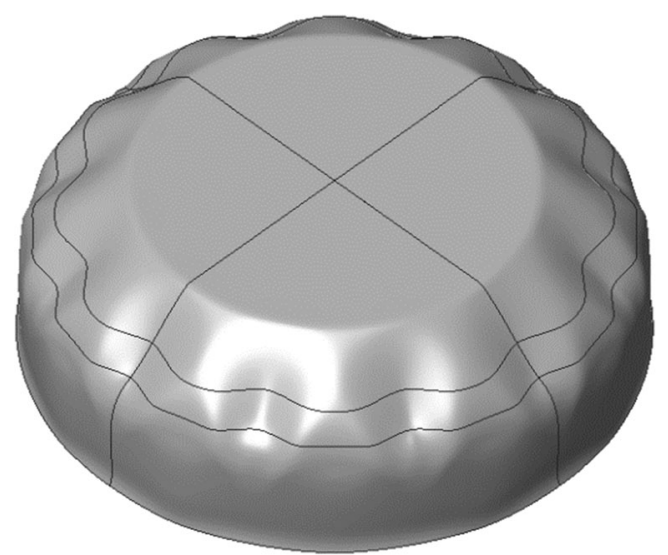

Fig. 6 Deformed geometry of the cylindrical cup predicted by mesh \#2 considering material isotropy (condition A)

The material anisotropy parameters obtained from this identification procedure are listed in Table 2 for the AA5042 aluminium alloy. The comparison between experimental and numerical results of the initial uniaxial yield stress values and the Lankford coefficients are presented in Fig. 4 (a) and (b), respectively. The set of material parameters obtained with DD3MAT provides a definition of the anisotropic yield surface in good agreement with the experimental values, particularly for the plastic strain ratio distribution.

\section{Mesh sensitivity analysis}

The in-plane mesh refinement of the blank has been identified by Kim et al. [12] as a numerical parameter that strongly influences the wrinkling prediction. Thus, the influence of mesh refinement on the numerical results is analysed using five different finite element meshes. The sensitivity finite element analysis is performed considering isotropic (von Mises) material behaviour before applying the advanced anisotropic yield criterion previously presented [34]. Only condition A was tested since the likelihood of wrinkling occurrence is higher in this condition than in condition $\mathrm{C}$, since the angle of the conical cup is lower [16]. Firstly, the in-plane mesh refinement is studied considering a fixed number of finite elements through the thickness (2 layers). After that, the number of elements along the thickness direction is analysed considering the in-plane mesh selected in the first stage. Concerning the structured zone of the mesh (see Fig. 3), the number of finite elements along the radial direction (NERD) and along the circumferential direction (NECD) are specified in Table 3, for the three meshes adopted in the in-plane mesh refinement study. The finite element length in radial direction and the central angle defined by the element in the structured zone are specified for each mesh. The total number of finite elements employed in each zone (structured and unstructured) of the blank is indicated, as well.

The punch force evolution with its displacement (full cup drawing) is shown in Fig. 5 (a) for each finite element mesh defined in Table 3. Since the region of the blank in contact with the rigid tools is contained within in the structured zone (concentric mesh), each circumferential line of blank nodes comes into contact simultaneously with the tools when considering isotropic material behaviour. Thus, small oscillations in the punch force evolution occur, particularly for the coarse mesh (mesh \#1), which presents a smaller number of finite elements in the radial direction (see Table 3). The punch force increases abruptly at around $8 \mathrm{~mm}$ of displacement because the largest cross section of the punch comes into contact with previously formed wrinkles (see Fig. 1). The deformed configuration of the cylindrical cup provided by mesh \#2 is presented in Fig. 6 assuming material isotropy (condition A). The side-wall wrinkling behaviour is evaluated in the present study through the radial distance of the cup after full drawing, measured in the two parallel cross sections indicated in Fig. 6, specifically at $z=-3.5 \mathrm{~mm}$ and $z=-5.5 \mathrm{~mm}$. Since the coordinate system origin is located on the top surface of the cup with outward orientation (see Fig. 1), the cross sections perpendicular to the cup axis are defined by negative values of $z$-coordinate. The radial coordinate of the drawn cup defined as function of the angular position is presented in Fig. 5 (b) for the finite element meshes specified in Table 3. Considering the coarse mesh (see mesh \#1 in Table 3), the finite element size adopted for the circumferential direction is insufficient to reproduce accurately the wrinkling waves (amplitude and frequency). Consequently, the punch displacement for which the punch force increases suddenly occurs later, defining the instant where the wrinkles touch the largest cross section of the punch. For both results analysed, only the coarse mesh (mesh \#1) leads to significant differences in comparison with the other two finite element meshes, as shown in Fig. 5.

The second study concerning the mesh sensitivity analysis deals with the number of finite elements adopted in the thickness direction. Since the numerical results are identical for mesh \#2 and mesh \#3 (see Fig. 5), and the number of finite elements is considerably higher in mesh \#3 (see Table 3 ), the second stage is carried out using the in-plane discretization corresponding to mesh \#2. Then, two additional finite element meshes are created to analyse the mesh refinement in the thickness direction, namely
Table 4 Number of finite elements used in three different meshes with the same in-plane discretization (NETD: number of elements in the thickness direction)

\begin{tabular}{lllllll}
\hline & NERD & NECD & NETD & Structured zone & Unstructured zone & Total of elements \\
\hline Mesh \#2 & 120 & 200 & 2 & 48,000 & 3802 & 51,802 \\
Mesh \#4 & 120 & 200 & 1 & 24,000 & 1901 & 25,901 \\
Mesh \#5 & 120 & 200 & 3 & 72,000 & 5703 & 77,703 \\
\hline
\end{tabular}




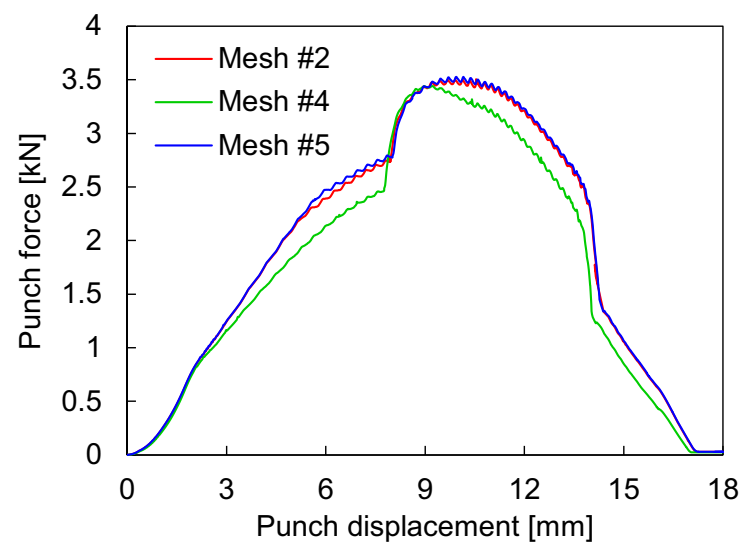

(a)

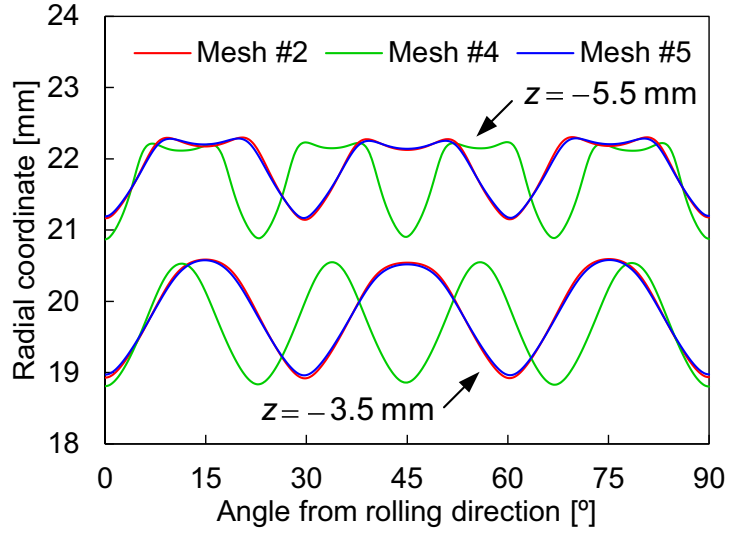

(b)

Fig. 7 Mesh refinement in the thickness direction considering material isotropy (condition A): (a) punch force evolution; (b) radial coordinate of the side-wall cup evaluated in two layers as function of the angular position

mesh \#4 (1 layer of finite elements) and mesh \#5 (3 layers of finite elements). The total number of finite elements composing each mesh is indicated in Table 4, as well as the number of elements employed in each direction of the structured zone (see Fig. 3).

The predicted punch force evolution is presented in Fig. 7 (a) for each finite element mesh indicated in Table 4 . The force value is considerably lower using the discretization with only 1 layer of finite elements through the thickness (mesh \#4). Furthermore, the punch displacement for which the force increases abruptly is slightly inferior adopting this mesh. The radial coordinate of the drawn cup as function of the angular position specified by the rolling direction is shown in Fig. 7 (b) for the three meshes in two parallel cross sections. The wrinkling pattern predicted by the mesh with only 1 layer of finite elements through the thickness presents a different number of wrinkling waves, although the height is similar. On the other hand, mesh \#2 and mesh \#5 provide identical results, both in terms of punch force evolution (Fig. 7 (a)) and wrinkling pattern (Fig. 7 (b)).

The computational performance of the numerical simulation using different finite element meshes is presented in Table 5. All numerical simulations are carried out on a computer machine equipped with an Intel® Core ${ }^{\mathrm{TM}}$ i7-2600 K Quad-Core processor (3.4 GHz) and the Windows 7 Professional (64-bits platform) operating system. The number of increments required to complete the forming process is between 706 and 748 . Therefore, the average increment size is approximately $0.025 \mathrm{~mm}$, while the average number of iterations per increment is about 8.5 . Note that the number of iterations required to achieve equilibrium in each increment is higher in drawing processes involving wrinkling than in classical sheet metal forming simulations, as shown in the deep drawing process of a cylindrical cup presented by Neto et al. [39]. The computational time increases exponentially with the mesh refinement, as shown in Table 5. In fact, the computational cost is about 3 days using the finer mesh (mesh \#3), while the adoption of mesh \#2 leads a computational time inferior to 1 day. Although the total number of finite elements composing the mesh \#3 and the mesh \#5 is similar (see Table 3 and Table 4), the computational cost is significantly lower adopting the mesh \#5 (3 layers of finite elements) since the number of contact elements is reduced. Comparing mesh \#2 with mesh \#5, the computational cost doubles when using 3 layers of finite elements through the thickness. Therefore, taking into account the computational time and the accuracy of the numerical results achieved, mesh \#2 is the discretization adopted in the following analysis.

\section{Results and discussion}

This section contains the comparison between experimental results and the numerical predictions for both conditions (punch $\mathrm{A}$ and punch $\mathrm{C}$ ), considering the anisotropic behaviour of the aluminium alloy described by the advanced yield criterion [34]. In order to quantify the effectiveness of the proposed finite element model, the punch force evolution and the shape of the wrinkles after the drawing process are evaluated in this study. The deformed configuration of the cylindrical cup after full drawing, predicted by the numerical model, is presented in Fig. 8 for both process conditions. The two cross sections
Table 5 Computational performance for five different finite element meshes considering material isotropy (condition A)

\begin{tabular}{llllll}
\hline & Mesh \#1 & Mesh \#2 & Mesh \#3 & Mesh \#4 & Mesh \#5 \\
\hline $\mathrm{N}^{\circ}$ of increments & 706 & 725 & 748 & 736 & 747 \\
$\mathrm{~N}^{\circ}$ of iterations/increment & 8.47 & 8.53 & 8.52 & 8.57 & 8.55 \\
Computational time [hours] & 7.4 & 22.7 & 69.8 & 13.3 & 45.0 \\
\hline
\end{tabular}


Fig. 8 Deformed geometry of the cylindrical cup after full drawing considering material anisotropy for: (a) condition A; (b) condition $\mathrm{C}$

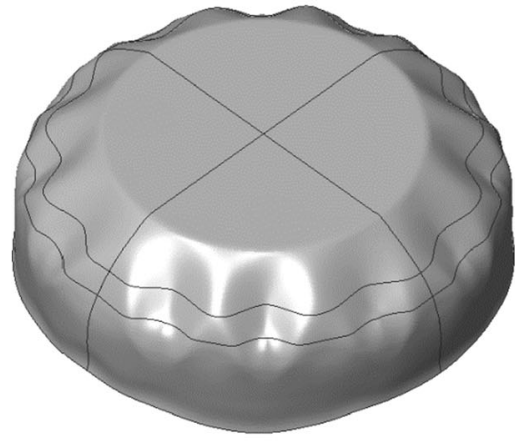

(a)

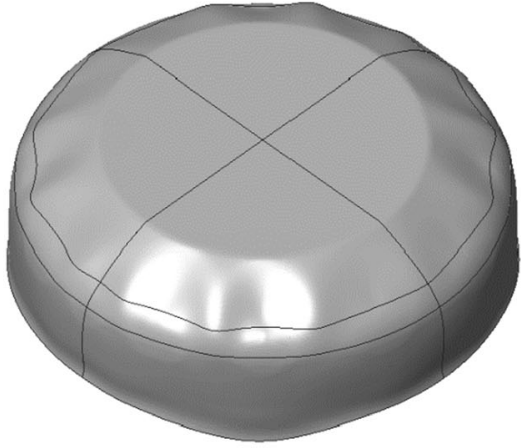

(b) selected to study the wrinkling behaviour are indicated in the figure, where the radial distance of the cup side-wall is calculated as function of the angular position specified by the rolling direction. The amplitude of the wrinkle pattern is strongly influenced by the tooling geometry (punch A and punch $\mathrm{C}$ ), increasing with the punch dimension $h$, as shown in Fig. 8.

\section{Punch force evolution}

The comparison between experimental and the numerically predicted punch force evolution is presented in Fig. 9 (a) and (b) for condition A and C, respectively. Since the reproducibility of the experimental punch force evolution is rather good, only one of the curves was chosen as representative of the results. The experimental punch force is overestimated by the numerical model for both conditions considered, as shown in Fig. 9. The modelling of the aluminium alloy using an isotropic work hardening law (Voce law given in Eq. (1)) can be the reason of this overestimation. In fact, typically the punch force value decreases when the kinematic hardening is taken into account, i.e. considering the Bauschinger effect
[40]. On the other hand, the influence of the material anisotropy in the punch force is negligible, as shown in Fig. 9.

The instant (punch displacement) in which the punch force increases abruptly is accurately predicted for both conditions, which occurs when the largest cross section of the punch comes into contact with formed wrinkles (see Fig. 1). Indeed, the punch force evolution (experimental and numerical) is identical for both conditions (punch A and punch C) until approximately $6 \mathrm{~mm}$ of punch displacement because the geometry of the punch in contact with the blank is the same (see Fig. 1). The abrupt drop of the experimental punch force that occurs at approximately $14.5 \mathrm{~mm}$ results from the loss of contact between the blank and the blank-holder. Since the experimental drawing system does not consider a rigid stop to keep the gap between the blank-holder and the die during the drawing process [16], the blank-holder promotes the material flow into the die cavity immediately before losing contact with the blank. This behaviour is not reproduced in the numerical simulation due to the stopping condition applied in the blank-holder, imposing that the gap between the blankholder and the die is always higher than $0.2 \mathrm{~mm}$.

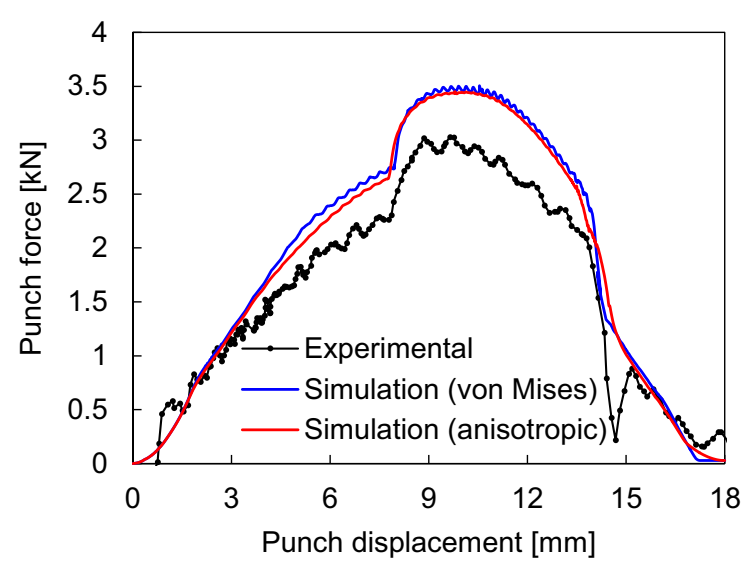

(a)

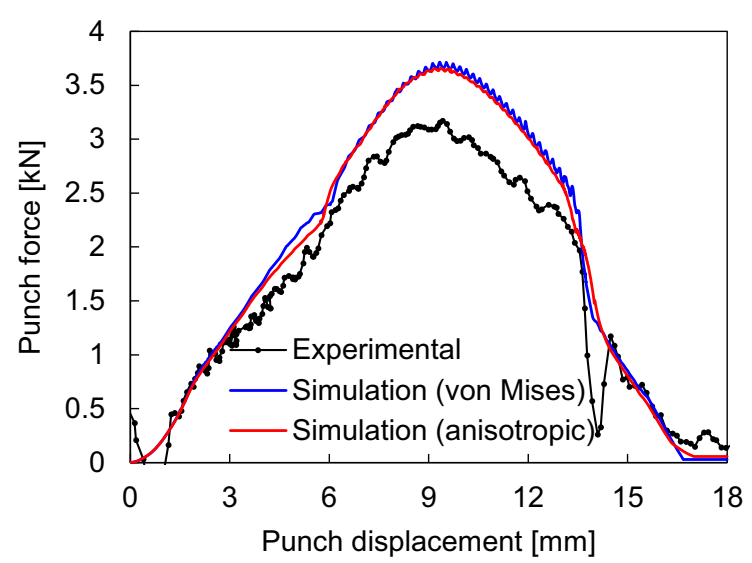

(b)

Fig. 9 Comparison between experimental and numerical punch force evolution for: (a) condition A; (b) condition C. Note that there is a small misprint in the conference proceedings [21] concerning the experimental punch force evolution for condition A 


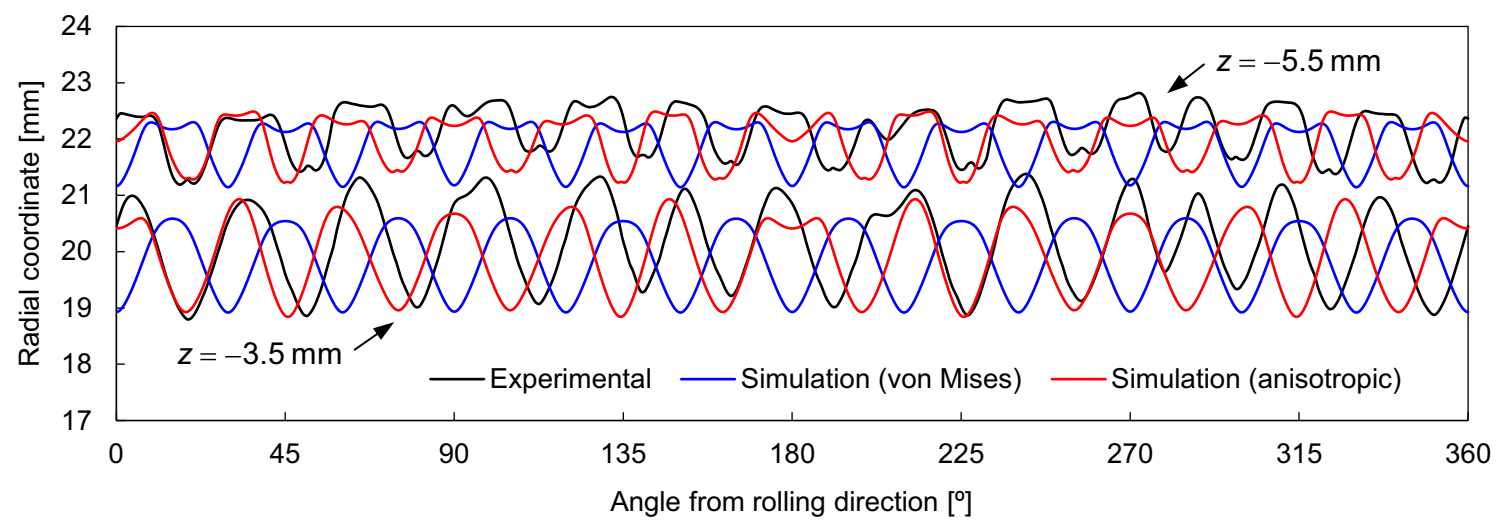

Fig. 10 Comparison between experimental and numerical radial coordinate of the side-wall cup measured in the planes $z=-3.5 \mathrm{~mm}$ and $z=-5.5 \mathrm{~mm}$ as function of the angular position (condition A)

\section{Wrinkle pattern}

The wrinkling behaviour is analysed after the full drawing process. The contour of the outer surface of the drawn cup is measured in two cross sections perpendicular to the cup axis $(z=-3.5 \mathrm{~mm}$ and $z=-5.5 \mathrm{~mm}$ ), which are illustrated in Fig. 8 . The comparison between experimental and numerical radial coordinate of the drawn cup, evaluated in these two layers, is presented in Fig. 10 for the process condition A. The number of the wrinkling waves observed experimentally is 13 in both cross sections, while the finite element model predicts 12 waves considering either material isotropy or anisotropy, as shown in Fig. 10. Since experimentally it is extremely difficult to ensure that the tools and blank are concentric, the wavelength is not constant along the circumferential direction. On the other hand, the numerical model takes advantage of the geometric and material symmetry conditions (one-quarter), providing always a wrinkling pattern with an even number of waves.

The constitutive behaviour of the aluminium alloy, namely the yield criterion adopted to describe the sheet anisotropy, has a large impact on the wrinkle prediction. The wrinkling pattern predicted with the anisotropic yield criterion Cazacu\&Barlat 2001 is substantially different from the one obtained considering material isotropy (von Mises), as shown in Fig. 10. In fact, considering the distribution of the cup radial coordinate as function of the angular position, each peak switches to a valley and vice-versa when the anisotropic yield criterion is adopted. Therefore, the advanced anisotropic yield criterion used in the present study provides numerical results that are in better agreement with the experimental results, as shown in Fig. 10. For both cross sections analysed, the amplitude of the wrinkling waves predicted by numerical simulation using the anisotropic yield criterion is similar to the one measured experimentally. Moreover, the small wrinkles arising in each valley of the experimental radial coordinate distribution for the cross section $z=-5.5 \mathrm{~mm}$ (see Fig. 10) are accurately predicted using the anisotropic yield criterion and overlooked considering isotropy.

The comparison between the experimental and numerical radial coordinate of the drawn cup is presented in Fig. 11 for the process condition $\mathrm{C}$. The amplitude of the wrinkling waves obtained with condition $\mathrm{C}$ is significantly lower than with condition A (see Fig. 10). Therefore, the decrease of the punch dimension $h$ (Fig. 1) decreases the likelihood of wrinkling occurrence. The radial coordinate of the drawn cup is slightly underestimated by the numerical model in both cross sections investigated, as shown in Fig. 11. The number of the wrinkling waves observed experimentally in the cross section closest to the top surface of the drawn

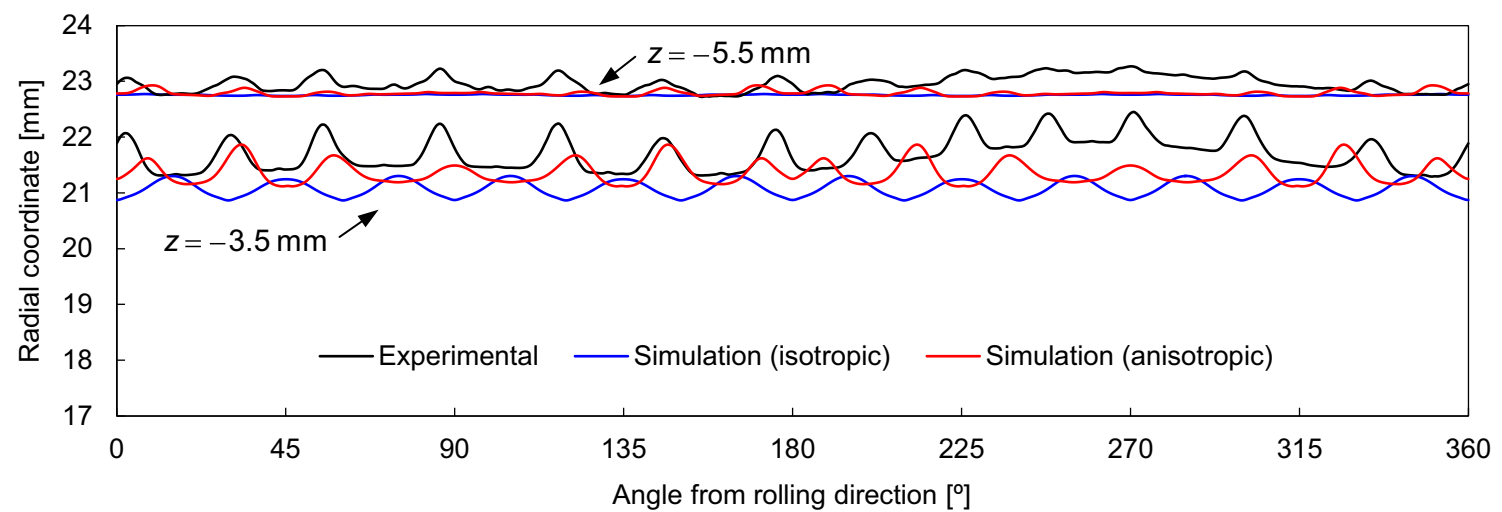

Fig. 11 Comparison between experimental and numerical radial coordinate of the side-wall cup measured in the planes $z=-3.5 \mathrm{~mm}$ and $z=-5.5 \mathrm{~mm}$ as function of the angular position (condition C) 


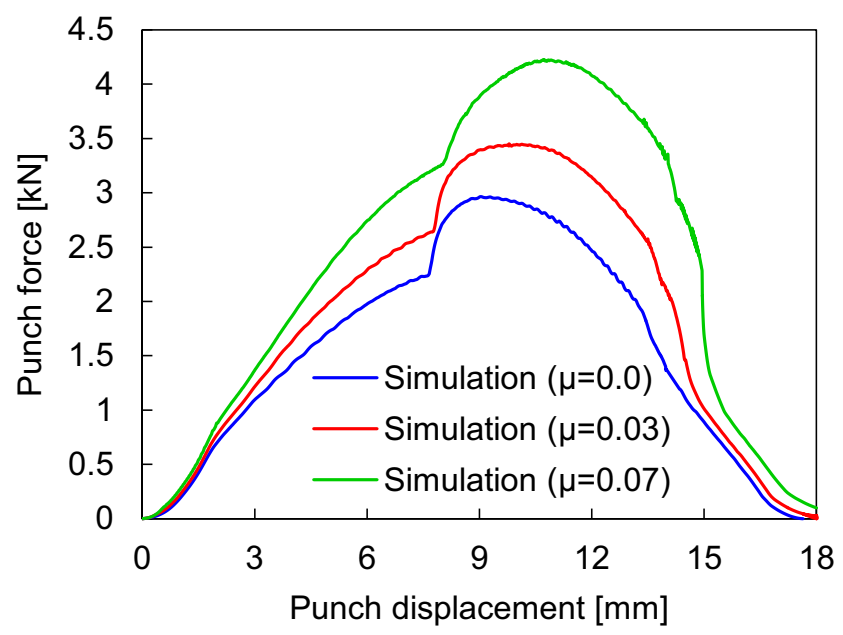

Fig. 12 Influence of the friction coefficient in the predicted punch force (condition A)

$\operatorname{cup}(z=-3.5 \mathrm{~mm})$ is 13 , while the finite element model yields 14 wrinkles considering material anisotropy, as shown in Fig. 11. On the other hand, considering material isotropy (von Mises), the finite element model predicts 12 wrinkles with lower amplitude. Furthermore, the angular location of the wrinkles is in complete disagreement with the experimental measurements. Concerning the cross section farthest from the top surface of the cup $(z=-5.5 \mathrm{~mm})$, the wrinkling tendency in the side-wall is relatively small, particularly for the numerical prediction. In fact, when assuming material isotropy the occurrence of wrinkling is imperceptible (amplitude lower than $0.02 \mathrm{~mm}$ ), as shown in Fig. 11.

Adopting the advanced anisotropic yield criterion, the shape of the wrinkles is accurately predicted in both cross sections. Nevertheless, its amplitude is lower in the numerical simulation than in the experimental measurement, mainly near the transverse direction of the sheet (see $90^{\circ}$ and $270^{\circ}$ with the rolling direction in Fig. 11). Since during deep drawing of cylindrical cups the sheet located between the blank-holder and die is subjected to uniaxial compression [41], the anisotropic behaviour of the aluminium alloy leads to a non-uniform thickening of the flange along the circumferential direction. The sheet thickening is higher near the transverse direction than in the rolling direction $[42,43]$ because the Lankford coefficient is smaller than 1.0 in the rolling direction and higher than 1.0 in the transverse direction (see Fig. 4 (b)). Therefore, the normal contact pressure and consequently the restraining blank holding force are higher in the transverse direction, which leads to lower wrinkling tendency. Indeed, the amplitude of the wrinkles arising in the transverse direction is lower than the ones predicted for the rolling direction, as shown in Fig. 11.

\section{Friction conditions}

The recommended value for the friction coefficient $\mu=0.03$, provided by the benchmark committee [21], was the one adopted in all numerical simulations previously presented. Nevertheless, the experimental measurement of the friction coefficient is difficult since it is influenced by several factors, such as the contact pressure, the sliding velocity, the surfaces roughness, the lubrication conditions, etc. [24]. Therefore, in the present study the side-wall wrinkling behaviour is evaluated for three constant values of friction coefficient $(0.0,0.03$ and 0.07 ). Figure 12 presents the predicted punch force evolution for the process condition A, assuming material anisotropy defined by the advanced yield criterion described in Section 3.1. As expected, the punch force value increases with the friction coefficient. Indeed, considering the higher value of friction coefficient $(0.07)$ the maximum value of punch force increases almost $40 \%$ in comparison with the frictionless contact conditions, as shown in Fig. 12. Therefore, this drawing process is very sensitive to the friction conditions, specifically to the magnitude of the contact forces in the flange.

The wrinkling behaviour evaluated through the radial coordinate of the drawn cup, measured in two cross sections, is presented in Fig. 13, for the three different values of friction coefficient. Since the value of the restraining forces arising in the flange increases with the friction coefficient, the amplitude of the wrinkling waves is lower for the higher value of friction coefficient (0.07), as shown in Fig. 13. Furthermore, the number of wrinkles arising in the cylindrical cup after full drawing increases from 12 to 14 when adopting the higher value of

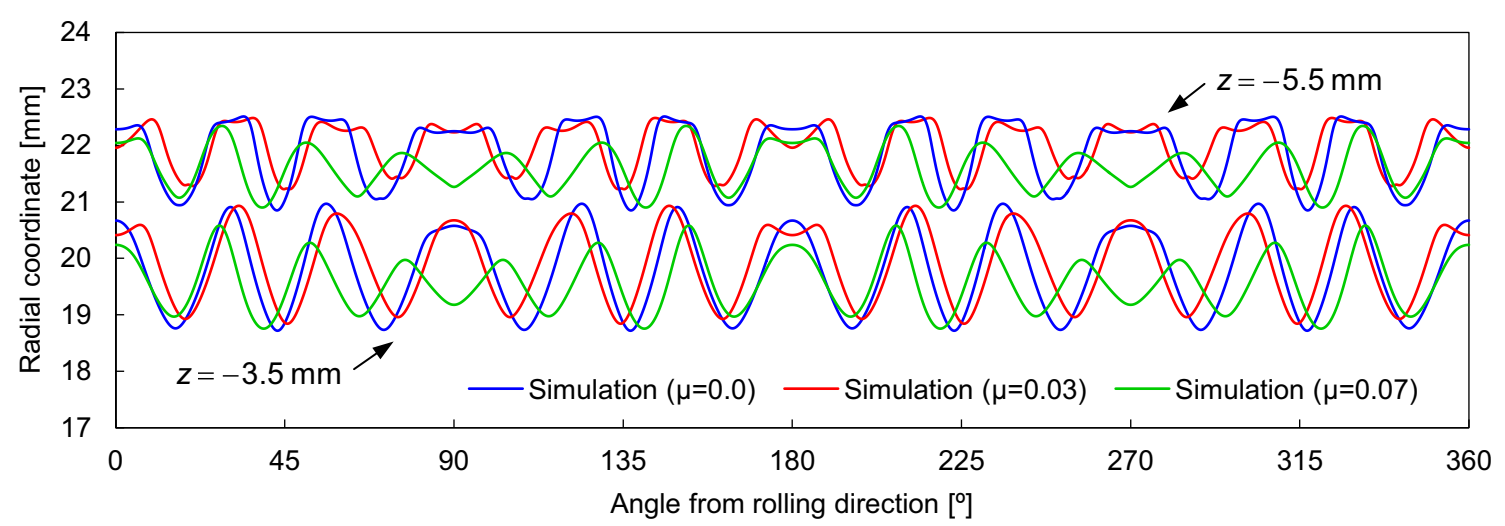

Fig. 13 Influence of the friction coefficient in the radial coordinate of the side-wall cup measured in the planes $z=-3.5 \mathrm{~mm}$ and $z=-5.5 \mathrm{~mm}$ (condition $\mathrm{A}$ ) 


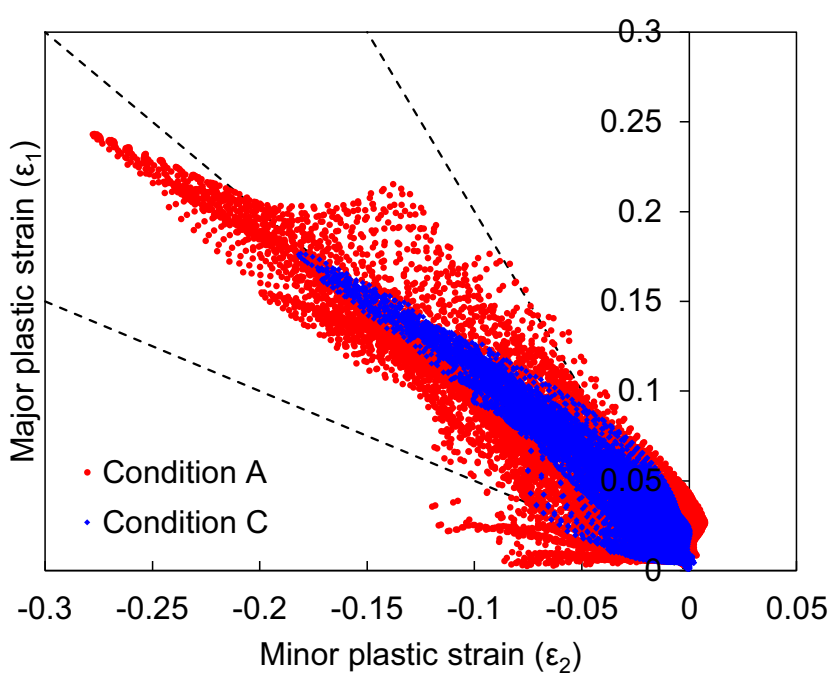

Fig. 14 Partial forming limit diagram with the minor-major strain plots for both process conditions after full drawing

friction coefficient. Regarding the shape and amplitude of the wrinkles, the difference between frictionless drawing and low friction coefficient value (0.03) is negligible, mainly in cross section $z=-3.5 \mathrm{~mm}$. Therefore, taking into account the experimental wrinkling profile (Fig. 10), the constant value of friction coefficient provided by the benchmark committee [21] seems to be appropriate to use in the numerical simulation.

\section{Strain distribution}

Both forming process conditions considered in this study (punch A and punch C) yield side-wall wrinkling in the cylindrical cup drawing of AA5042 aluminium alloy. Nevertheless, the amplitude of the wrinkles is significantly lower for condition $\mathrm{C}$, in comparison with the condition $\mathrm{A}$, as highlighted in the final deformed configuration of the aluminium cups predicted by numerical simulation (Fig. 8). The wrinkling phenomena occurs when the minor stress in the sheet is compressive. Therefore this failure mode can be identified in the tension-compression domain of the forming limit diagram (FLD), which is defined by positive major and negative minor strains. Considering isotropic material behaviour (von Mises), the pure shear strain path is defined by $\varepsilon_{1}=-\varepsilon_{2}$, and the uniaxial tension strain path by $\varepsilon_{2}=-0.5 \varepsilon_{1}$, while the strain path referent to uniaxial compression is expressed by $\varepsilon_{2}=-2 \varepsilon_{1}$. Figure 14 presents for both process conditions the partial forming limit diagram after full cup drawing, evaluated only for the nodes located in the outer surface of the cup side-wall. The minor-major plastic strain distribution of these nodes is mainly positioned around the pure shear strain path (assuming isotropy). However, the dispersion is higher in condition A than in condition C (see Fig. 14), which varies essentially from uniaxial compression to uniaxial tension. Moreover, the maximum value of equivalent plastic strain predicted in the cup side-wall is higher for the condition A.

The representation of the partial forming limit diagram in the deformed cup is shown in Fig. 15 for both conditions. The forming limit diagram is divided in four zones according to the ratio between minor and major strains. The safe forming region is defined for strain paths between uniaxial tension and full biaxial stretching, while the wrinkling tendency occurs for strain paths between uniaxial tension and pure shear. On the other hand, the severe wrinkle region is defined by strain paths varying from uniaxial compression to plane strain (compressive), while the wrinkle zone is located between uniaxial compression and pure shear.

The occurrence of bending in the circumferential direction (wrinkles) results from the compressive stress in the same direction. For both process conditions studied, the side-wall area of the cup is subjected to strain paths related with wrinkling and severe wrinkling. Besides, this area is considerably larger in condition A than in condition C, as shown in Fig. 15. In fact, the amplitude of the wrinkles arising in the conical side-wall of the drawn cup is significantly higher for condition A (compare in Fig. 10 with Fig. 11). The magnitude of the minor plastic strain is higher in the valley of the wrinkles than in the peak due to the bending effects in the side-wall. Therefore, the material state predicted through the FLD is severe wrinkling and safe for the

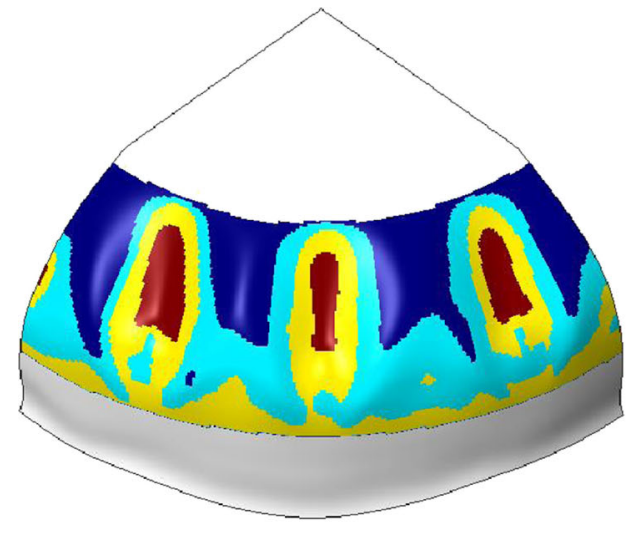

(a)

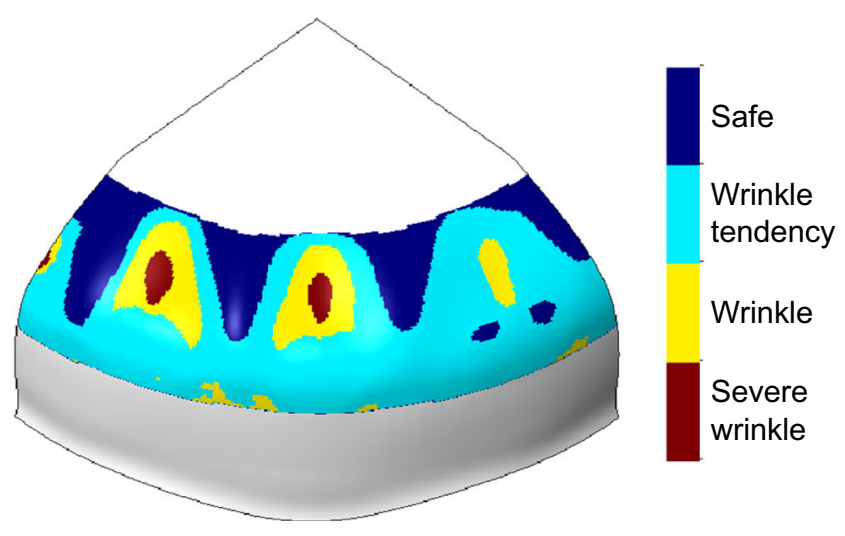

(b)

Fig. 15 Different zones of the partial forming limit diagram plotted on the deformed cup for: (a) condition A; (b) condition C 
valley and peak of the wrinkles, respectively. The wrinkling tendency during cup drawing can be accurately predicted using the FLD provided by the finite element analysis. Considering the example under analysis, both process conditions are susceptive to wrinkling, nevertheless the likelihood of occurrence is higher for the condition A (see zones with severe wrinkle in Fig. 15).

\section{Conclusions}

The occurrence of side-wall wrinkling during the sheet metal forming process is investigated by the finite element method using the non-bifurcation analysis. The benchmark 4 proposed in the Numisheet 2014 conference - Wrinkling during cup drawing - is the example selected for this study. Two different process operating conditions are analysed and compared, which differ only in the punch geometry. The numerical prediction of the wrinkling behaviour in the cylindrical cup drawing process is compared with experimental results. The overall influence of the plastic anisotropy modelling on the wrinkling tendency is evaluated by two different yield criteria. Accordingly, the behaviour of the AA5042 aluminium alloy is described considering material isotropy (von Mises) as well as adopting an advanced yield criterion (Cazacu\&Barlat 2001).

The numerical results, namely the punch force evolution and the shape of the formed wrinkles, are very sensitive to the blank mesh. Therefore, the selection of the finite element mesh for the blank is a key point for an accurate prediction of the wrinkling in the drawn cup. Indeed, the in-plane mesh used to predict the wrinkling behaviour must be significantly finer than the one employed to simulate the classical drawing process in absence of wrinkles. The experimental punch force evolution is slightly overestimated by the numerical model for both process conditions considered. On the other hand, applying the advanced yield criterion to describe the aluminium anisotropy, the side-wall wrinkling behaviour is accurately predicted by the finite element model, specifically the shape, number and amplitude of the wrinkles. Nevertheless, the selection of the yield criterion to model the material anisotropy induces significant changes in the wrinkling. In fact, the locations of the peak and the valley in the wrinkling profile are reversed when the aluminium alloy is modelled as an isotropic material. Since the presented numerical model takes into account the real process conditions, namely the geometry of the forming tools and the frictional contact conditions with the blank, the numerical results are in good agreement with the experimental ones.

\footnotetext{
Acknowledgments The authors gratefully acknowledge the financial support of the Portuguese Foundation for Science and Technology (FCT) under project PTDC/EMS-TEC/1805/2012. The first author is also grateful to the FCT for the Postdoctoral grant SFRH/BPD/101334/2014 and P.D. Barros is grateful to the FCT for the PhD Grant SFRH/BD/98545/2013.
}

\section{References}

1. Banabic D (2010) Sheet metal forming processes: constitutive modelling and numerical simulation. Springer, Berlin. doi:10. 1007/978-3-540-88113-1

2. Pham CH, Thuillier S, Manach PY (2014) Twisting analysis of ultra-thin metallic sheets. J Mater Process Technol 214:844-855. doi:10.1016/j.jmatprotec.2013.12.006

3. Kim S-H (2007) Improvement of the surface quality of an automotive member by a modification of the stamping tool. J Mater Process Technol 187-188:387-391. doi:10.1016/j.jmatprotec. 2006.11.210

4. Anarestani SS, Morovvati MR, Vaghasloo YA (2015) Influence of anisotropy and lubrication on wrinkling of circular plates using bifurcation theory. Int J Mater Form 8:439-454. doi:10.1007/ s12289-014-1187-6

5. Schwindt CD, Bertinetti MA, Iurman L, et al. (2015) Numerical study of the effect of martensite plasticity on the forming limits of a dual-phase steel sheet. Int J Mater Form. doi:10.1007/s12289-0151236-9

6. Dick RE, Yoon JW (2015) Wrinkling during cup drawing with NUMISHEET2014 benchmark test. Steel Res Int. doi:10.1002/ srin. 201500018

7. Shafaat MA, Abbasi M, Ketabchi M (2011) Investigation into wall wrinkling in deep drawing process of conical cups. J Mater Process Technol 211:1783-1795. doi:10.1016/j. jmatprotec.2011.05.026

8. Wang C-T, Kinzel G, Altan T (1994) Wrinkling criterion for an anisotropic shell with compound curvatures in sheet forming. Int J Mech Sci 36:945-960. doi:10.1016/0020-7403(94)90056-6

9. Wang X, Cao J (2000) On the prediction of side-wall wrinkling in sheet metal forming processes. Int J Mech Sci 42:2369-2394. doi: 10.1016/S0020-7403(99)00078-8

10. Agrawal A, Reddy NV, Dixit PM (2007) Determination of optimum process parameters for wrinkle free products in deep drawing process. J Mater Process Technol 191:51-54. doi:10.1016/j. jmatprotec.2007.03.050

11. Kim JB, Yang DY (2003) Prediction of wrinkling initiation in sheet metal forming processes. Eng Comput 20:6-39. doi:10.1108/ 02644400310458810

12. Kim JB, Yoon JW, Yang DY (2003) Investigation into the wrinkling behaviour of thin sheets in the cylindrical cup deep drawing process using bifurcation theory. Int J Numer Methods Eng 56:1673-1705. doi:10.1002/nme.629

13. Yu TX, Johnson W (1982) The buckling of annular plates in relation to the deep-drawing process. Int J Mech Sci 24:175-188. doi: 10.1016/0020-7403(82)90036-4

14. Riks E (1979) An incremental approach to the solution of snapping and buckling problems. Int J Solids Struct 15:529-551. doi:10. 1016/0020-7683(79)90081-7

15. Cao J, Boyce MC (1997) Wrinkling behavior of rectangular plates under lateral constraint. Int J Solids Struct 34:153-176. doi:10. 1016/S0020-7683(96)00008-X

16. Kawka M, Olejnik L, Rosochowski A, et al. (2001) Simulation of wrinkling in sheet metal forming. J Mater Process Technol 109: 283-289. doi:10.1016/S0924-0136(00)00813-X

17. Henriques MP, de Sousa RJA, Valente RAF (2010) Numerical implicit strategies for wrinkling prediction in free and flange forming of anisotropic sheets. Int J Mater Form 3:907-910. doi:10.1007/ s12289-010-0915-9

18. Kim J, Kang S-J, Kang B-S (2003) A comparative study of implicit and explicit FEM for the wrinkling prediction in the hydroforming process. Int J Adv Manuf Technol 22:547-552. doi:10.1007/ s00170-003-1540-2 
19. Isik K, Silva MB, Tekkaya AE, Martins PAF (2014) Formability limits by fracture in sheet metal forming. J Mater Process Technol 214:1557-1565. doi:10.1016/j.jmatprotec.2014.02.026

20. Kim Y, Son Y (2000) Study on wrinkling limit diagram of anisotropic sheet metals. J Mater Process Technol 97:88-94. doi:10. 1016/S0924-0136(99)00346-5

21. Dick RE, Cardoso R, Paulino M, Yoon JW (2013) Benchmark 4 wrinkling during cup drawing. AIP Conf Proc 1567:262-327. doi: $10.1063 / 1.4849984$

22. ASTM-A681 (2008) Standard Specification for Tool Steels Alloy. doi: $10.1520 / a 0681-08$

23. Menezes LF, Teodosiu C (2000) Three-dimensional numerical simulation of the deep-drawing process using solid finite elements. J Mater Process Technol 97:100-106. doi:10.1016/S0924-0136(99) 00345-3

24. Oliveira MC, Alves JL, Menezes LF (2008) Algorithms and strategies for treatment of large deformation frictional contact in the numerical simulation of deep drawing process. Arch Comput Methods Eng 15:113-162. doi:10.1007/s11831-008-9018-x

25. Neto DM, Oliveira MC, Menezes LF, Alves JL (2014) Applying Nagata Patches to smooth discretized surfaces used in 3D frictional contact problems. Comput Methods Appl Mech Eng 271:296-320. doi:10.1016/j.cma.2013.12.008

26. Neto DM, Oliveira MC, Menezes LF, Alves JL (2013) Nagata patch interpolation using surface normal vectors evaluated from the IGES file. Finite Elem Anal Des 72:35-46. doi:10.1016/j.finel.2013.03. 004

27. Alart P, Curnier A (1991) A mixed formulation for frictional contact problems prone to newton like solution methods. Comput Methods Appl Mech Eng 92:353-375. doi:10.1016/0045-7825(91)90022-X

28. Yamada Y, Yoshimura N, Sakurai T (1968) Plastic stress-strain matrix and its application for the solution of elastic-plastic problems by the finite element method. Int J Mech Sci 10:343-354. doi:10. 1016/0020-7403(68)90001-5

29. Menezes LF, Neto DM, Oliveira MC, Alves JL (2011) Improving computational performance through HPC techniques: case study using DD3IMP in-house code. AIP Conf Proc 1353:1220-1225. doi:10.1063/1.3589683

30. Hughes TJR (1980) Generalization of selective integration procedures to anisotropic and nonlinear media. Int J Numer Methods Eng 15:1413-1418. doi:10.1002/nme.1620150914

31. Teodosiu C, Daniel D, Cao H-L, Duval J-L (1995) Modelling and simulation of the can-making process using solid finite elements. J Mater Process Technol 50:133-143. doi:10.1016/0924-0136(94) 01375-B
32. Moreira L, Ferron G, Ferran G (2000) Experimental and numerical analysis of the cup drawing test for orthotropic metal sheets. J Mater Process Technol 108:78-86. doi:10.1016/S0924-0136(00)00660-9

33. Barlat F, Maeda Y, Chung K, et al. (1997) Yield function development for aluminum alloy sheets. J Mech Phys Solids 45:17271763. doi:10.1016/S0022-5096(97)00034-3

34. Cazacu O, Barlat F (2001) Generalization of Drucker's yield criterion to orthotropy. Math Mech Solids 6:613-630. doi:10.1177/ 108128650100600603

35. Flores P, Tuninetti V, Gilles G, et al. (2010) Accurate stress computation in plane strain tensile tests for sheet metal using experimental data. J Mater Process Technol 210:1772-1779. doi:10.1016/ j.jmatprotec.2010.06.008

36. Chaparro BM, Thuillier S, Menezes LF, et al. (2008) Material parameters identification: gradient-based, genetic and hybrid optimization algorithms. Comput Mater Sci 44:339-346. doi:10.1016/j. commatsci.2008.03.028

37. Soare S, Yoon JW, Cazacu O (2008) On the use of homogeneous polynomials to develop anisotropic yield functions with applications to sheet forming. Int J Plast 24:915-944. doi:10.1016/j. ijplas.2007.07.016

38. Barros PD, Simões VM, Neto DM, et al. (2013) On the influence of the yield parameters identification procedure in cylindrical cups earing prediction. AIP Conf Proc. 1567:512-515. doi:10.1063/1. 4850024

39. Neto DM, Oliveira MC, Alves JL, Menezes LF (2014) Comparing faceted and smoothed tool surface descriptions in sheet metal forming simulation. Int J Mater Form. doi:10.1007/s12289-014$1177-8$

40. Oliveira MC, Alves JL, Chaparro B, Menezes LF (2007) Study on the influence of work-hardening modeling in springback prediction. Int J Plast 23:516-543. doi:10.1016/j.ijplas.2006.07.003

41. Neto DM, Oliveira MC, Alves JL, Menezes LF (2014) Influence of the plastic anisotropy modelling in the reverse deep drawing process simulation. Mater Des 60:368-379. doi:10.1016/j.matdes. 2014.04.008

42. Yoon JW, Dick RE, Barlat F (2011) A new analytical theory for earing generated from anisotropic plasticity. Int J Plast 27:11651184. doi:10.1016/j.ijplas.2011.01.002

43. Chung K, Kim D, Park T (2011) Analytical derivation of earing in circular cup drawing based on simple tension properties. Eur J Mech - A/Solids 30:275-280. doi:10.1016/j.euromechsol. 2011.01.006 